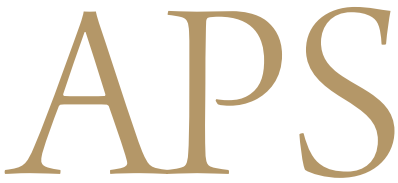

Archives of Plastic Surgery

\title{
Cervicofacial Lymphatic Malformations: A Retrospective Review of 40 Cases
}

\author{
Byung Chae Cho ${ }^{1}$, Jae Bong Kim ${ }^{1}$, Jeong Woo Lee ${ }^{1}$, Kang Young Choi ${ }^{1}$, Jung Dug Yang ${ }^{1}$, \\ Seok-Jong Lee ${ }^{2}$, Yong-Sun $\mathrm{Kim}^{3}$, Jong Min Lee ${ }^{3}$, Seung Huh ${ }^{4}$, Ho Yun Chung ${ }^{1}$ \\ Departments of ${ }^{1}$ Plastic and Reconstructive Surgery, ${ }^{2}$ Dermatology, ${ }^{3}$ Radiology, and ${ }^{4}$ Vascular Surgery, Kyungpook National University \\ School of Medicine, Daegu, Korea
}

Background Lymphatic malformation (LM) is a form of congenital vascular malformation with a low incidence. Although LM has been studied, no consensus has emerged regarding its cause or treatment.

Methods In this study, we retrospectively evaluated 40 patients who visited our vascular anomalies center for the treatment of cervicofacial LM, which is a common manifestation of LM. The medical records of patients over a period of 12 years were reviewed and analyzed for commonalities regarding the diagnosis and the results of treatment.

Results Suspected cervicofacial LM was confirmed through imaging studies. No difference in incidence was observed according to sex, and 73\% of patients first presented with symptoms before the age of two years. The left side and the V2-V3 area were most commonly affected. No significant differences in incidence were observed among the macrocystic, microcystic, and combined types of LM. A total of 28 out of 36 patients received sclerotherapy as the first choice of treatment, regardless of the type of lesion. Complete resolution was achieved in only $25 \%$ of patients.

Conclusions LM is important to confirm the diagnosis early and to choose an appropriate treatment strategy according to the stage of the disease and each individual patient's symptoms. When treatment is delayed or an incorrect treatment is administered, patient discomfort increases as the lesion gradually spreads. Therefore, more so than is the case for most other diseases, a team approach on a case-by-case basis is important for the accurate and appropriate treatment of LM.

Keywords Lymphatic abnormalities / Lymphangioma / Sclerotherapy

\author{
Correspondence: Ho Yun Chung \\ Department of Plastic and \\ Reconstructive Surgery, Kyungpook \\ National University School of \\ Medicine, 130 Dongdeok-ro, Jung-gu, \\ Daegu 41944, Korea \\ Tel: +82-53-420-5692 \\ Fax: +82-53-425-3879 \\ E-mail: hy-chung@knu.ac.kr
}

This study was supported by the Basic Science Research Program through the National Research foundation of Korea (NRF) funded by the Ministry of Education (NRF-2014R1A1A4A01009584).

This research was supported by the Kyungpook National University Research Fund, 2012.

No potential conflict of interest relevant to this article was reported.

\section{INTRODUCTION}

Lymphatic malformation (LM) is a form of congenital vascular malformation that stems from the malformation of the lymphatic vessels in soft tissues, including the skin [1]. Since the nineteenth century, LM has been known as cystic hygroma and lym- phangioma, and classified as a tumor of lymphatic origin. Mulliken and Glowacki [2] coined the currently preferred term, lymphatic malformation, in their 1982 classification of vascular anomalies.

LM has a slow flow and low incidence, and its causes have not been clearly delineated. van der Putte and van Limborgh [3] 
have argued that the lymphatic system originates and develops from the venous system at six weeks of gestation; at nine to ten weeks gestation, the lymphatic system of the entire body starts to grow and form connections throughout the body. Considerable support exists for the theory that LM occurs when sequestered lymphatic tissue fails to communicate with the lymphatic and venous systems after budding during the developmental process of the normal lymphatic system $[1,4]$.

Possibly due to embryological factors, LM occurs more commonly together with other vascular malformations, such as capillary malformation and venous malformation, than it does alone, which makes it difficult to establish the precise incidence of $L M$. However, some authors have argued that its incidence is approximately $2.8-5$ out of 100,000 people $[5,6]$. Moreover, symptoms of LM are observed immediately after birth in more than $50 \%$ of patients and are observed before the age of two years in $90 \%$ of patients [7].

Histologically, LM is classified into the macrocystic type, consisting of cysts larger than $2 \mathrm{~cm}$ with clear boundaries (previously known as cystic hygromas), and the microcystic type, consisting of cysts smaller than $2 \mathrm{~cm}$ that appear diffuse and sprouted without clear boundaries (previously known as lymphangio$\mathrm{ma})$. The two types coexist in the combined type $[2,8,9]$.

LM can occur anywhere in the lymphatic system, but previous research has indicated that up to $75 \%$ of cases of LM manifest as cervicofacial lesions (in the tongue, lip, cheek, or neck), as these are regions where the lymphatic system predominates $[8,10,11]$. The macrocystic type has been most frequently reported to appear in the neck area, while the microcystic type has been reported to appear most frequently in the intraoral cavity and cheek area.

Unlike LM in other regions, cervicofacial LM can cause airway obstruction and become life-threatening. Since cosmetic sequelae and functional impairment are possible complications of the surgical treatment of LM, it is important to administer early treatment after an accurate diagnosis and to choose appropriate methods according to the location and depth of the lesion.

The objective of the present study was to analyze the clinical features, diagnostic process, treatment methods, and treatment results for patients with cervicofacial LM.

\section{METHODS}

This study included 40 patients who were collaboratively diagnosed with cervicofacial LM by specialists from the fields of plastic surgery, dermatology, radiology, and vascular surgery. We then retrospectively reviewed the patients' medical histories. We excluded cases accompanied by primary lymphedema, other types of vascular anomalies, or congenital cysts, such as branchial cleft cysts or thyroglossal duct cysts.

Based on each patient's medical history, we determined the age at the first presentation of symptoms, the age at the patient's first visit to the treatment center, sex, and average follow-up period. The distribution of $\mathrm{LM}$ was classified into the left, center, or right side of the head and neck, and into the V1, V2, and V3 areas. The location of $\mathrm{LM}$ was classified into 10 regions, including the scalp and forehead, ear, cheek, and intraoral locations. When LM was present in more than two regions, we obtained the number of cysts in each location. Moreover, LM was classified as macrocystic, microcystic, or a combined type in which both types coexisted, using a cut-off value of $2 \mathrm{~cm}$ for distinguishing between the macrocystic and microcystic types $[2,12]$.

A team of specialists conducted a physical examination in all patients with suspected vascular malformations. Magnetic resonance imaging (MRI) $[13,14]$ and Doppler ultrasonography (D-USG) $[15,16]$ were used to confirm the diagnosis of patients with suspected LM. The treatment team gathered and discussed the type and location of LM, as well as cosmetic factors, in order to decide on a course of treatment. Sclerotherapy, surgical resection, and $\mathrm{CO}_{2}$ laser ablation using OK-432 and bleomycin were the primary courses of initial treatment, and depending on the situation, more than two types of treatment methods were used sequentially. D-USG and the presence of residual symptoms, which can be grossly confirmed through a physical examination, were used to evaluate the extent to which the LM lesions decreased in size and to classify the therapeutic effect as aggravated, partially improved ( $<25 \%)$, improved ( $>50 \%$ ), or completely improved ( $>90 \%)$.

\section{RESULTS}

\section{Clinical features}

A total of 1,752 patients who visited our treatment center over a period of 12 years were diagnosed with vascular malformations. Of these patients, 149 (8.5\%) had pure LM, excluding mixed types of vascular malformations, such as lymphaticovenous malformation. Forty patients $(2.3 \%)$ had a cervicofacial lesion. The study participants included 21 males and 19 females-an approximately 1:1 ratio-and no significant difference in incidence was observed with respect to sex. The average age at the first presentation of symptoms in the patients was $4.47 \pm 8.82$ years old (range, 0 month-30 years) for males and $5.26 \pm 10.43$ years old (range, 0 month-46 years) for females. A total of 21 patients (53\%) showed symptoms at birth, including 10 males (48\%) and 11 females (58\%). A total of eight patients (four males, four females) showed symptoms after birth but before the age of two 
years. The average follow-up period was $61.2 \pm 79.25$ months (range, 1-325 months), including four patients who were lost to follow-up.

\section{Type, distribution, and location}

Based on the initial physical examination and imaging studies of patients with suspected cervicofacial vascular anomalies, we evaluated the type of LM present in each patient as well as its distribution and location. Thirteen patients had macrocystic

\section{Fig. 1. Type of cervicofacial lymphatic malformation}

Types of cervicofacial lymphatic malformations, with no significant differences observed according to type.

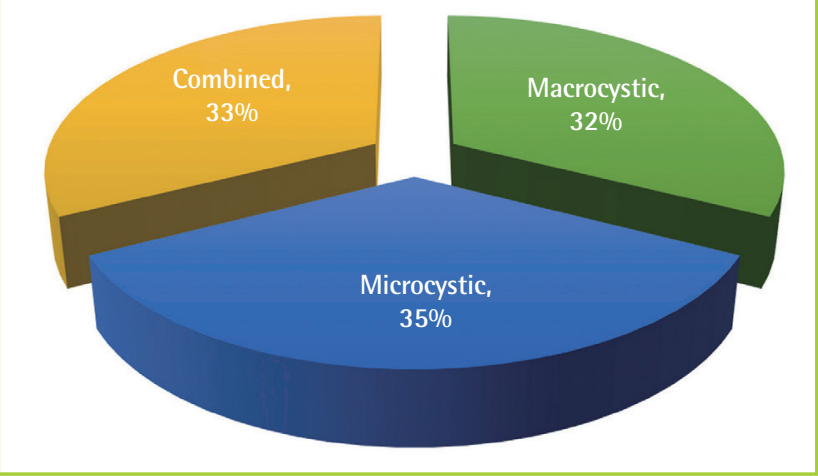

\section{Fig. 2. Distribution of cervicofacial lymphatic malformations}

The left side and the $\mathrm{V} 2-\mathrm{V} 3$ areas were the predominant regions.
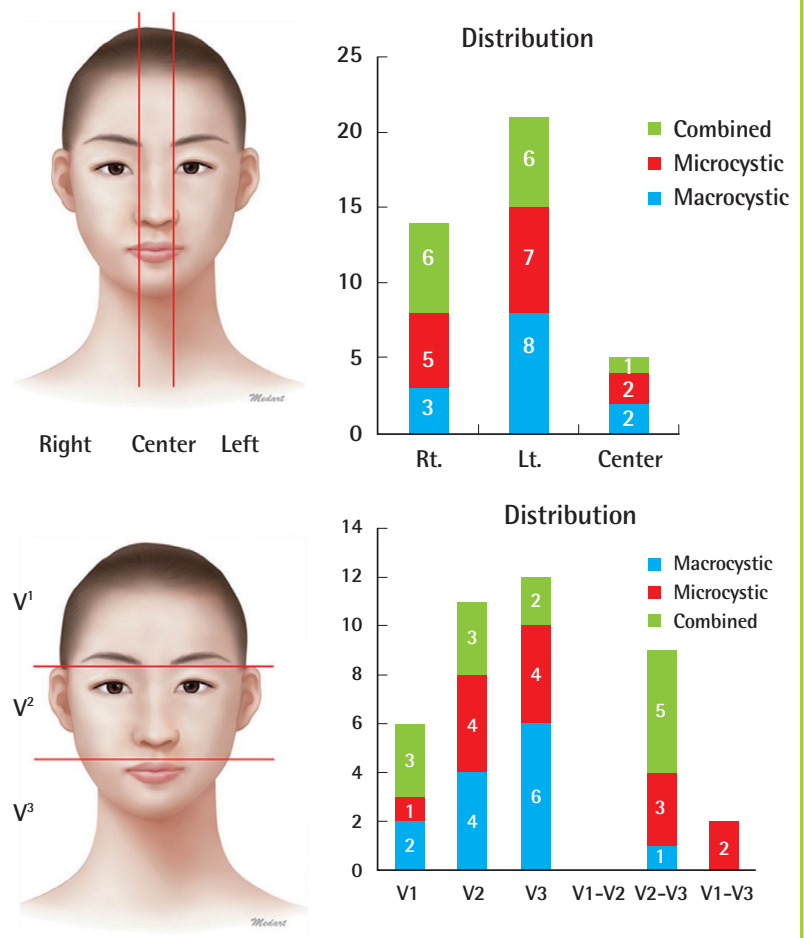

LM (32.5\%), 14 patients had microcystic LM (35\%), and 13 patients had combined LM, and these differences were not statistically significant (Fig. 1).

Fourteen patients (35\%) had right-side lesions, 21 patients (53\%) had left-side lesions, and five patients (12\%) had center lesions. Six patients (15\%) had V1 lesions, 11 patients (28\%) had V2 lesions, and 12 patients (30\%) had V3 lesions. Among the patients with LM in more than two areas, nine $(23 \%)$ had V2-V3 lesions and two (5\%) had V1-V3 lesions (Fig. 2). No significant differences were observed in the distribution of lesions according to the type of LM.

We then categorized the lesions depending on whether they occurred in the scalp and forehead, eyelid, orbital cavity, ear, cheek (parotid area), nose, lip, mandible, neck, or intraoral area.

\section{Fig. 3. Location of cervicofacial lymphatic malformations}

The areas with the most lesions were the cheek (19 patients), mandible (13 patients), and neck (10 patients).

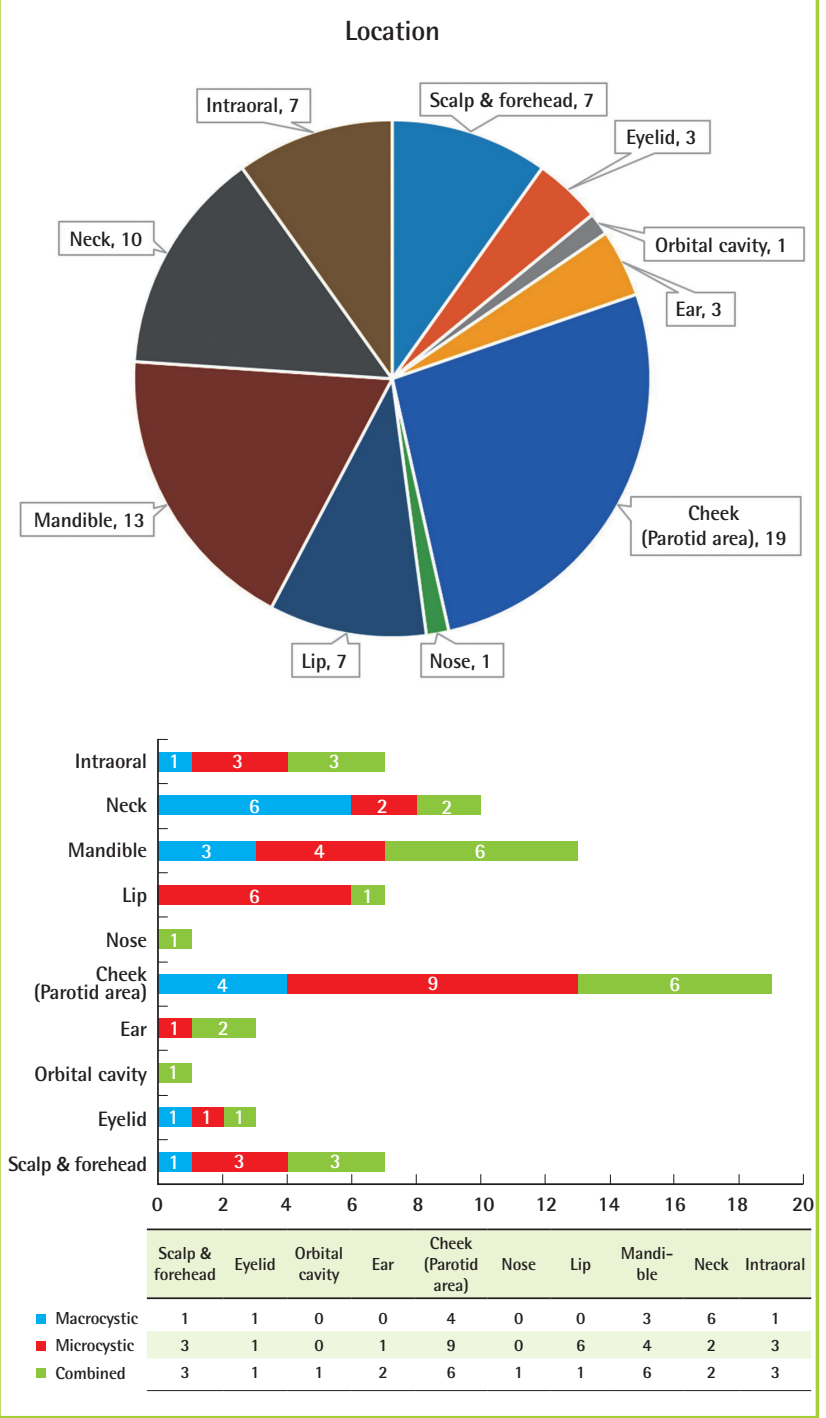


The highest frequency was observed in the cheek (parotid area) (19 patients, $47.5 \%$ ), mandible (13 patients, $32.5 \%$ ), and neck (10 patients, 25\%). Fifteen patients (37.5\%) showed a widespread distribution, with LM present in at least two regions. The macrocystic type was commonly found in the neck area and the microcystic type was commonly found in the cheek (parotid area) and lip. The combined type, however, showed an even distribution over the cervicofacial region (Fig. 3).

\section{Diagnosis}

After taking a medical history from patients with suspected vascular anomalies and conducting a physical examination, LM was confirmed using imaging studies such as D-USG, which is noninvasive and relatively accessible, or MRI, which allows subtypes to be identified and the extent of the lesion and of invasion into nearby soft tissues to be determined. Thirty-three patients (82.5\%) underwent D-USG and 36 patients (90\%) underwent MRI. Due to the burden of radiation, computed tomography was only performed when the size of the lesion and its relationships with adjacent structures needed to be clarified, and was administered to only 15 patients (37.5\%). The diagno- sis was confirmed by biopsy in 13 patients, including those who underwent surgical resection during the treatment process.

\section{Treatment}

The treatment strategies used included sclerotherapy, surgical excision, and simple passive observation, and the treatment methods included $\mathrm{CO}_{2}$ laser ablation, $\mathrm{OK}-432$, or bleomycin. Of the 40 patients diagnosed with cervicofacial LM, four were lost to follow-up and two showed complete improvement after six months of follow-up.

Of the 12 patients with macrocystic LM, four (33\%) received sclerotherapy more than once, and three of the 13 patients with microcystic LM (23\%) received sclerotherapy more than once as well as surgical excision. Of the 11 patients with combinedtype LM, three (27\%) received sclerotherapy once and four (36\%) received sclerotherapy more than once. Of the 36 patients who were diagnosed with LM and were followed up at our treatment center, a total of 28 (78\%) received sclerotherapy at least once, regardless of the type of LM.

Improvement or complete improvement was observed in eight of the 12 patients (67\%) with macrocystic LM, nine of the 13

\section{Table 1. Treatment methods and outcomes of cervicofacial lymphatic malformations according to type $(n=40)$}

\begin{tabular}{|c|c|c|c|c|c|c|c|c|}
\hline Tyре & \multicolumn{2}{|c|}{ Treatment } & $\begin{array}{l}\text { Partially } \\
\text { improved }\end{array}$ & Improved & $\begin{array}{l}\text { Completely } \\
\text { improved }\end{array}$ & Aggravated & & Total \\
\hline \multirow[t]{9}{*}{ Macrocystic } & \multicolumn{2}{|l|}{ Passive observation } & 0 & 0 & 1 & 0 & 1 & 12+1 loss to follow-up \\
\hline & \multicolumn{2}{|l|}{ Sclerotherapy } & 0 & 1 & 1 & 0 & 2 & \\
\hline & \multirow[t]{2}{*}{ Sclerotherapy } & Sclerotherapy & 0 & 2 & 1 & 1 & 4 & \\
\hline & & $\mathrm{OP}$ & 0 & 0 & 0 & 0 & 0 & \\
\hline & $\mathrm{OP}$ & & 0 & 0 & 1 & 0 & 1 & \\
\hline & \multirow[t]{2}{*}{$\mathrm{OP}$} & $\mathrm{OP}$ & 1 & 0 & 0 & 0 & 1 & \\
\hline & & Sclerotherapy & 1 & 1 & 0 & 0 & 2 & \\
\hline & \multicolumn{2}{|c|}{$\mathrm{CO}_{2}$ laser ablation } & 1 & 0 & 0 & 0 & 1 & \\
\hline & $\mathrm{CO}_{2}$ laser ablation & Sclerotherapy & 0 & 0 & 0 & 0 & 0 & \\
\hline \multirow[t]{8}{*}{ Microcystic } & \multicolumn{2}{|c|}{ Sclerotherapy } & 0 & 2 & 0 & 0 & 2 & $13+1$ loss to follow-up \\
\hline & \multirow[t]{2}{*}{ Sclerotherapy } & Sclerotherapy & 0 & 3 & 0 & 0 & 3 & \\
\hline & & $\mathrm{OP}$ & 1 & 0 & 0 & 0 & 1 & \\
\hline & $\mathrm{OP}$ & & 0 & 0 & 2 & 0 & 2 & \\
\hline & \multirow[t]{2}{*}{$\mathrm{OP}$} & $\mathrm{OP}$ & 1 & 1 & 0 & 1 & 3 & \\
\hline & & Sclerotherapy & 0 & 0 & 0 & 0 & 0 & \\
\hline & \multicolumn{2}{|c|}{$\mathrm{CO}_{2}$ laser ablation } & 0 & 0 & 1 & 0 & 1 & \\
\hline & $\mathrm{CO}_{2}$ laser ablation & Sclerotherapy & 1 & 0 & 0 & 0 & 1 & \\
\hline \multirow[t]{9}{*}{ Combined } & \multicolumn{2}{|c|}{ Passive observation } & 0 & 0 & 1 & 0 & 1 & $11+2$ lost to follow-up \\
\hline & \multicolumn{2}{|l|}{ Sclerotherapy } & 0 & 2 & 0 & 1 & 3 & \\
\hline & \multirow[t]{2}{*}{ Sclerotherapy } & Sclerotherapy & 0 & 1 & 1 & 2 & 4 & \\
\hline & & $\mathrm{OP}$ & 0 & 0 & 0 & 0 & 0 & \\
\hline & $\mathrm{OP}$ & & 0 & 0 & 0 & 0 & 0 & \\
\hline & \multirow[t]{2}{*}{$\mathrm{OP}$} & $\mathrm{OP}$ & 0 & 1 & 0 & 0 & 1 & \\
\hline & & Sclerotherapy & 0 & 1 & 0 & 1 & 2 & \\
\hline & \multicolumn{2}{|l|}{$\mathrm{CO}_{2}$ laser ablation } & 0 & 0 & 0 & 0 & 0 & \\
\hline & $\mathrm{CO}_{2}$ laser ablation & Sclerotherapy & 0 & 0 & 0 & 0 & 0 & \\
\hline \multicolumn{3}{|c|}{ 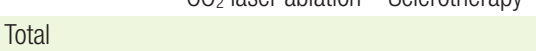 } & 6 & 15 & 9 & 6 & 36 & 40 (including those lost to follow-up) \\
\hline
\end{tabular}


patients (69\%) with microcystic LM, and seven of the 11 patients $(63 \%)$ with combined-type LM. Similar trends in improvement were noted in all three types of LM. However, aggravation despite continuous treatment was observed in one patient (8\%) with macrocystic LM, one patient (7\%) with microcystic LM, and four patients (36\%) with combined-type LM, showing a clear difference among the three conditions. Since combinedtype LM was associated with a higher likelihood of aggravation despite continuous treatment, including surgical resection and sclerotherapy, many patients experienced difficulty after treatment (Table 1).

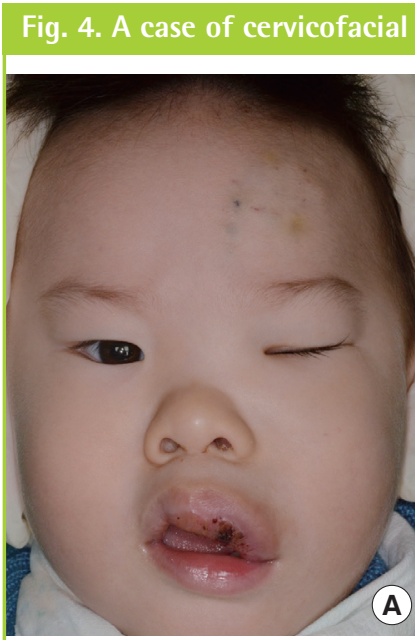

(A) A pre-treatment photo of a child with LM, showing swelling in the upper lip and eyelid area. (B) Pre-treatment MRI, T2 phase, with the orange arrows indicating the high signal intensity of the LM lesion. (C) Pre-treatment D-USG image, where the red circle indicates a combined-type LM lesion. (D) Photo of the patient at four years of age, showing aggravated swelling, pain, and symptoms. (E) Post-sclerotherapy D-USG image, where the red circle indicates a sprouting LM channel. (F) Sclerotherapy procedure using bleomycin. LM, lymphatic formation; MRI, magnetic resonance imaging; D-USG, Doppler ultrasonography.
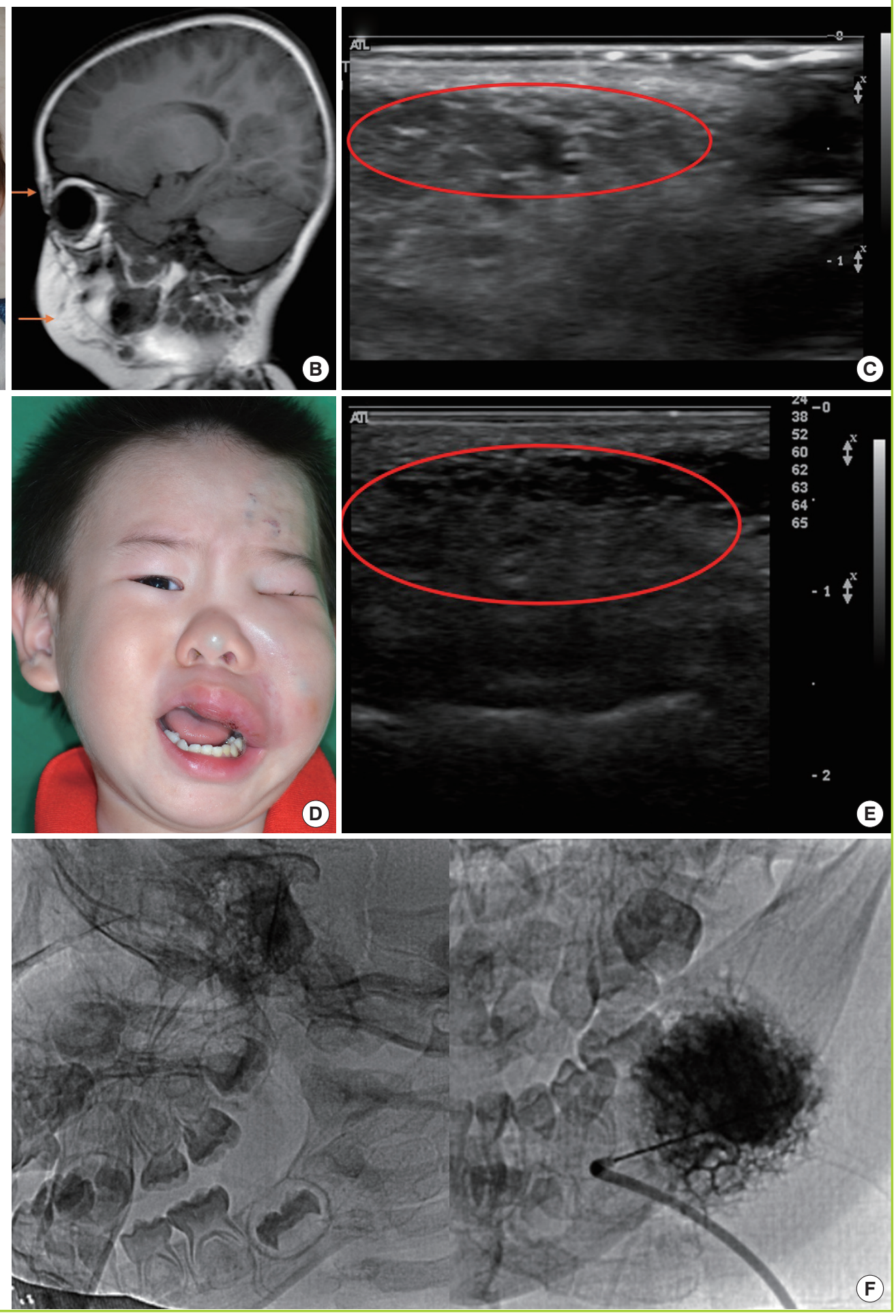


\section{Case 1}

A child who exhibited swelling in the upper lip and eyelid at birth visited our treatment center for blue- and red-tinged patches and swelling on the left side of the hemifacial area as well as ulceration and bleeding in the intraoral area. At the time of the ini- tial diagnosis, a medical history was taken and the physical examination took place during a team conference. After an imaging study using D-USG and MRI, the child was diagnosed with combined-type LM (Fig. 4A-C). In order to treat and prevent infection, bleeding, and ulceration, the patient underwent $\mathrm{CO}_{2}$

Fig. 5. A case of cervicofacial lymphatic malformation
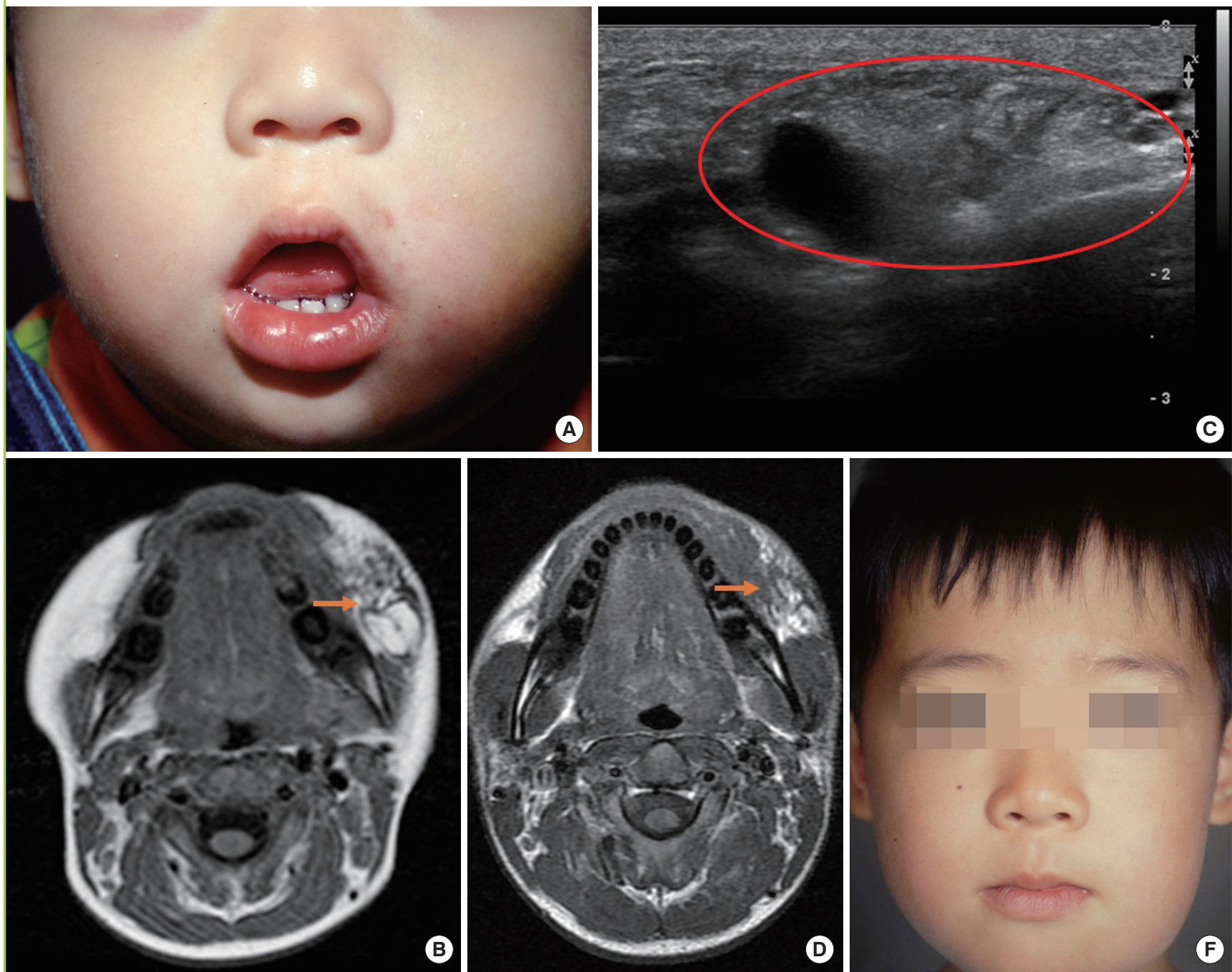

(A) A pre-treatment photo of a child with $L M$ who presented with swelling on the left cheek. (B) Pre-treatment MRI, T2 phase, with the orange arrow indicating the high signal intensity of the combinedtype LM lesion. (C) Pre-treatment D-USG image, where the red circle indicates the combined-type LM lesion. (D) Postoperation MRI, T2 phase, where the orange arrow indicates the high signal intensity of the microcystic LM lesion. (E) Postoperation D-USG image, where the red circle indicates the remnant microcystic LM lesion. (F) Photo of the patient at seven years of age; after resection, the macrocystic LM improved, but the microcystic LM remained present. LM, lymphatic formation; MRI, magnetic resonance imaging; D-USG, Doppler ultrasonography.

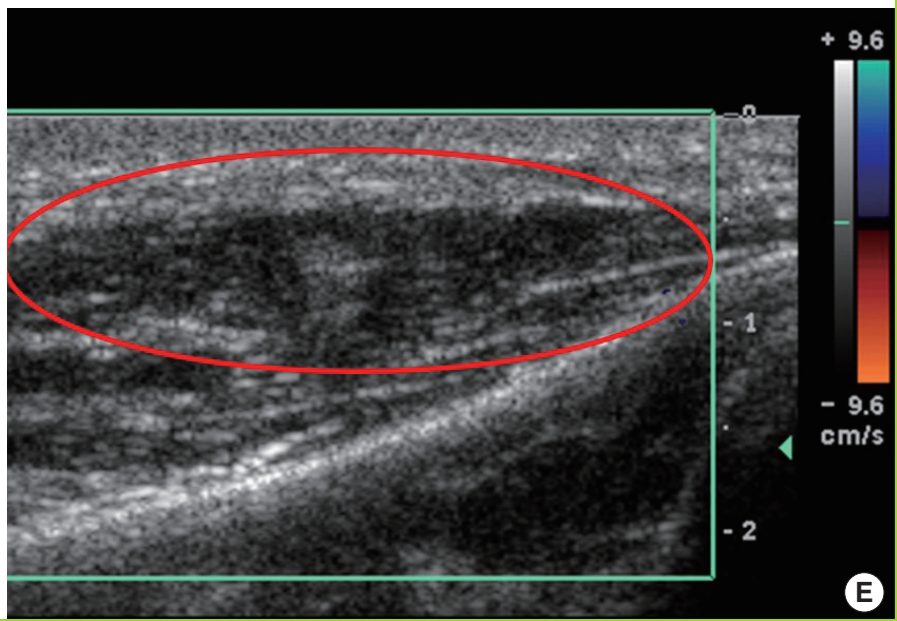


laser ablation and showed improvement. The patient also experienced swelling, aggravation, and pain in his forehead and eyelid. Sclerotherapy using bleomycin was administered, which alleviated the symptoms, and the patient underwent continual follow-up after the initial diagnosis. Two years later, the child again experienced swelling, aggravation, and pain in the areas of the previous forehead lesion and the eyelid, and underwent a second round of sclerotherapy using bleomycin (Fig. 4D-F). Appropriate treatment methods were chosen to address changes in the patient's condition according to the consensus of our center's vascular anomalies team.

\section{Case 2}

A child presented with swelling on the left cheek area at birth and visited our institution for a palpable mass observed in the same area six months after birth, as well as for increased mass size and swelling, facial asymmetry, and aggravation. After the medical history and physical examination were completed, the medical team diagnosed the patient with combined-type LM after completing an imaging study with MRI and D-USG (Fig. $5 \mathrm{~A}-\mathrm{C})$. The child underwent surgical resection to remove the macrocystic mass and the symptoms improved. Although follow-up observations using D-USG confirmed the removal of macrocystic LM, the microcystic LM remained and additional sclerotherapy was performed (Fig. 5D, E). No interference with the child's daily activity was observed. Based on the findings of improvement in the microcystic LM lesions using D-USG, continuous follow-up was planned with the understanding that the appropriate treatment method for any changes in the condition of the patient would be chosen based on the consensus of the center's vascular anomalies team (Fig. 5F).

\section{Case 3}

A woman presented with swelling on the right cheek area and visited our institution for a palpable mass observed in the same area, as well as for increased mass size and swelling, facial asym-

\section{Fig. 6. A case of cervicofacial lymphatic malformation}

(A) A pre-treatment photo of a woman with LM who experienced swelling on the right cheek. (B) Pre-treatment MRI, T2 phase, with the orange arrow indicating the high signal intensity of the microcystic LM lesion. (C) Pre-treatment D-USG image, where the green box indicates the microcystic LM lesion. (D) A post-sclerotherapy photo showing that the LM had completely improved. (E) A post-sclerotherapy D-USG image, where the green box shows that almost the entire LM lesion had been removed. LM, lymphatic formation; MRI, magnetic resonance imaging; D-USG, Doppler ultrasonography.
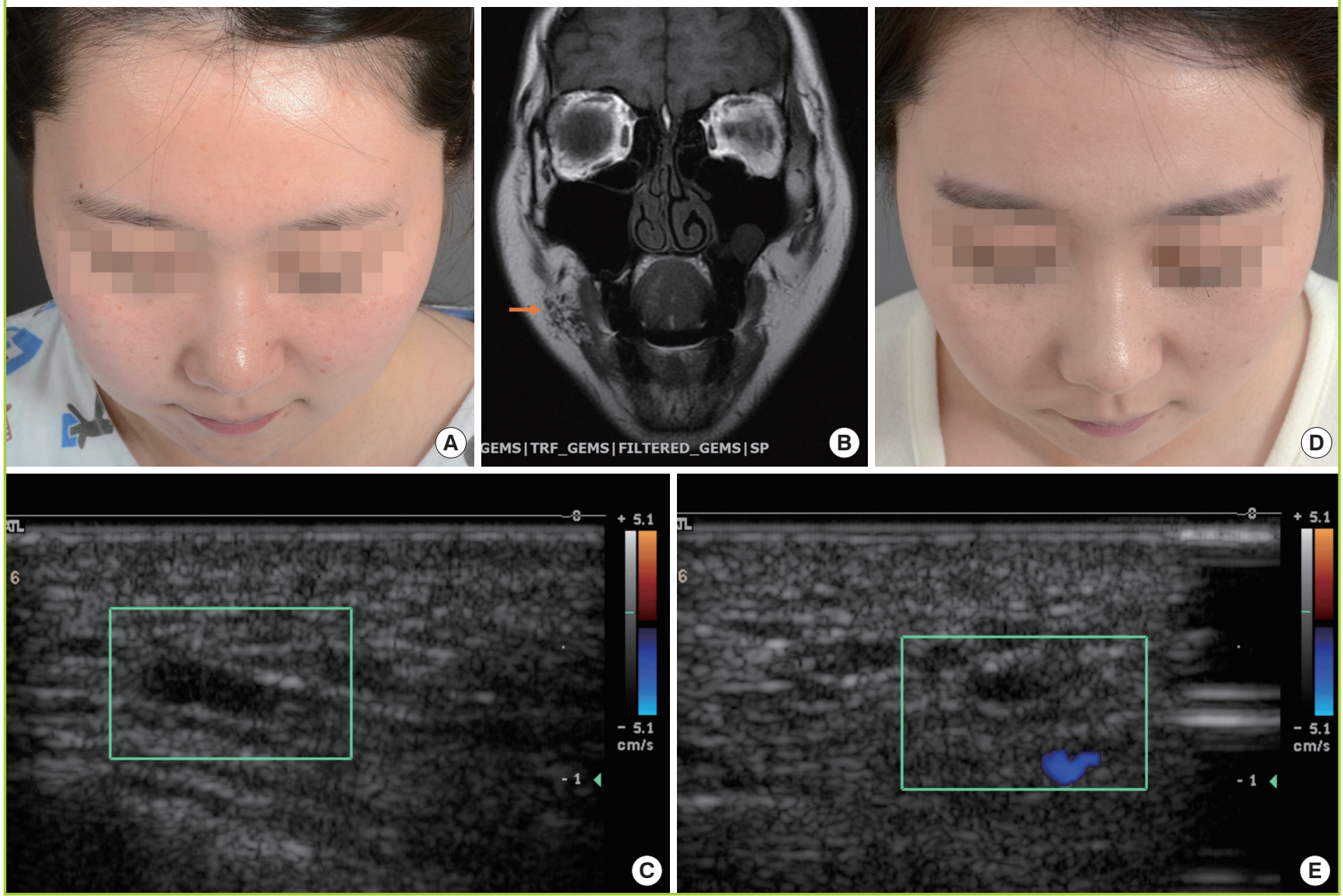
metry, and aggravation. After the medical history and physical examination were completed, the medical team diagnosed the patient with microcystic LM after completing an imaging study with MRI and D-USG (Fig. 6A-C). The woman underwent sclerotherapy using bleomycin to remove the microcystic mass and her symptoms were shown to improve by D-USG. (Fig. 6D, E). Since her microcystic LM was found to have completely improved, the plan was to perform continuous follow-up and to choose the appropriate treatment method for any changes in the condition of the patient according to the consensus of the center's vascular anomalies team.

\section{DISCUSSION}

Many studies, including research by Padwa and Mulliken, have established that LM is a congenital vascular malformation [7,12$14,17]$.

In agreement with the extant literature, we found that $52.5 \%$ of cases (21 patients) presented with symptoms at birth, and that patients who presented with symptoms before the age of two years accounted for $72.5 \%$ of cases (29 patients). In patients who presented with symptoms after the age of two years, LM was located in deep soft tissue lesions, and the symptoms were initially not observable with the naked eye. However, as the size of the lesion increased or the lesion sprouted diffusely due to an event such as trauma or infection, symptoms began to arise and patients visited the hospital. We observed a 1:1 sex ratio, which is consistent with the results of previous studies that have demonstrated no relationship between the incidence of LM and sex $[2,8,9,14]$.

As mentioned above, considerable support exists for the theory that LM occurs during the formation of the venous and lymphatic system at six to ten weeks of gestation, when sequestered lymphatic tissue fails to communicate with the lymphatic and venous system $[1,4]$. Therefore, LM generally manifests as cervicofacial lesions in areas where the lymphatic system first forms, most notably in the parotid area (cheek), mandible, and neck area, which account for a large part of the lymphatic system $[8$, $11,17,18]$. The results of our study also indicated that $80 \%$ of cases (32 patients) occurred in the $\mathrm{V} 2-\mathrm{V} 3$ region, which includes the cheek, intraoral area, mandible, and neck (Fig. 3).

The clinical features found in this study were similar to those reported by previous research. However, Greene [19] and Bisdorff et al. [20] reported that LM on the left side occurred in $62 \%$ to $70 \%$ of cases, while other authors have argued that LM has an equal incidence on the left and right side, and still other studies have reported opposing views regarding left-right disparities in the affected site. In the present study, $52.5 \%$ of cases
(21 patients) had LM on the left side, compared to 35\% of cases (14 patients) that were diagnosed with LM on the right side (Fig. 2). Studies of large patient groups have not been conducted to date, and thus few theories have been proposed regarding the cause of LM. The lymphatic system itself predominates on the left side, which we believe may be a possible reason for our findings $[20,21]$.

An accurate medical history and physical examination are important for diagnosing patients with vascular anomalies. Accordingly, in our vascular anomalies center, history taking and physical examinations are performed collaboratively at a weekly conference by a team of specialists from each related department. Imaging studies are then designed to focus on the suspected diagnosis. Based on the characteristics of the lesions in the cervicofacial region, which has ample soft tissue and contains many important organs, the size and form of LM can be confirmed. However, D-USG, which is often the first choice for diagnosing vascular anomalies, is often ineffective for identifying LM in deep soft tissue. Therefore, computed tomography must be performed selectively, taking into account the average age of the patients and their vulnerability to radiation. Consequently, MRI is the first-choice imaging modality for LM at our institution. In our study, 36 patients (90\%) underwent MRI. Taking into account the cost benefits and ease of accessibility during pregnancy, 33 patients (82.5\%) received additional testing using D-USG. Furthermore, D-USG was the modality most commonly used to observe the therapeutic effect of treatment during follow-up. Similarly to the present study, other authors have adopted the strategy of first obtaining a medical history and conducting a physical examination, then designing a treatment strategy after using MRI and D-USG to confirm the diagnosis and classification of LM [1,13,22-24].

Cervicofacial LM, as indicated by many studies, is a form of vascular malformation that is difficult to eradicate. Various suggestions for treatment methods have been made, including laser ablation, surgical resection, and sclerotherapy, depending on the type and location of $\mathrm{LM}[8,9,18,25]$. Of the 36 patients in our study who received treatment, 18 patients $(50 \%)$ received sclerotherapy only. Surgical resection can be performed only when LM is located in an accessible area, meaning that only eight patients $(22 \%)$ underwent surgical resection with sclerotherapy. Sclerotherapy was used as first choice of treatment regardless of the type of LM. Surgical resection was employed primarily for macrocystic LM, which forms cysts with clear boundaries, meaning that surgical resection poses no risk of damage to the neighboring structures. It was also used to a limited extent as a method for reducing the range of cosmetic side effects after treatment in some cases of microcystic and combined-type LM. 
Of the 36 patients, excluding four patients who were lost to follow-up, nine (25\%) showed complete improvement, 15 (42\%) showed improvement, six (17\%) showed partial improvement, and six (17\%) showed aggravation. Taking into account the high risk of recurrence of LM and the difficulty of eradication, these therapeutic outcomes may be interpreted as positive.

The diagnosis, examination, and choice of a treatment method are important factors in the treatment of various types of vascular anomalies, and this is even more imperative for types of vascular anomalies with no established standard protocol. Since a general diagnosis and treatment protocol has not been established for LM, a multi-faceted approach is required for each case. Based on 12 years of experience, we argue that the proper treatment method should involve a team of specialists from plastic surgery, pediatrics, dermatology, radiology, and vascular surgery, and that treatment should be planned on a case-by-case basis instead of following a standardized protocol.

\section{REFERENCES}

1. Mulliken JB, Burrows PE, Fishman SJ, et al. Mulliken and Young's vascular anomalies: hemangiomas and malformations. Oxford: Oxford University Press; 2013.

2. Mulliken JB, Glowacki J. Hemangiomas and vascular malformations in infants and children: a classification based on endothelial characteristics. Plast Reconstr Surg 1982;69: 412-22.

3. van der Putte SC, van Limborgh J. The embryonic development of the main lymphatics in man. Acta Morphol Neerl Scand 1980;18:323-35.

4. Fonkalsrud EW. Lymphatic disorders. In: Grosfeld JL, editor. Pediatric surgery. Philadelphia: Mosby/Elsevier; 2006. p.2137-46.

5. de Serres LM, Sie KC, Richardson MA. Lymphatic malformations of the head and neck: a proposal for staging. Arch Otolaryngol Head Neck Surg 1995;121:577-82.

6. Smith RJ. Lymphatic malformations. Lymphat Res Biol 2004; 2:25-31.

7. Wiggs WJ Jr, Sismanis A. Cystic hygroma in the adult: two case reports. Otolaryngol Head Neck Surg 1994;110:23941.

8. Ethunandan M, Mellor TK. Haemangiomas and vascular malformations of the maxillofacial region: a review. Br J Oral Maxillofac Surg 2006;44:263-72.

9. Kaban LB, Mulliken JB. Vascular anomalies of the maxillofacial region. J Oral Maxillofac Surg 1986;44:203-13.

10. Curran AJ, Malik N, McShane D, et al. Surgical management of lymphangiomas in adults. J Laryngol Otol 1996;110:586-9.

11. Kang GC, Song C. Forty-one cervicofacial vascular anomalies and their surgical treatment: retrospection and review. Ann Acad Med Singapore 2008;37:165-79.

12. Emery PJ, Bailey CM, Evans JN. Cystic hygroma of the head and neck: a review of 37 cases. J Laryngol Otol 1984;98: 613-9.

13. Barnes PD, Burrows PE, Hoffer FA, et al. Hemangiomas and vascular malformations of the head and neck: MR characterization. AJNR Am J Neuroradiol 1994;15:193-5.

14. Meyer JS, Hoffer FA, Barnes PD, et al. Biological classification of soft-tissue vascular anomalies: MR correlation. AJR Am J Roentgenol 1991;157:559-64.

15. Eivazi B, Fasunla AJ, Hundt W, et al. Low flow vascular malformations of the head and neck: a study on brightness mode, color coded duplex and spectral Doppler sonography. Eur Arch Otorhinolaryngol 2011;268:1505-11.

16. Griauzde J, Srinivasan A. Imaging of vascular lesions of the head and neck. Radiol Clin North Am 2015;53:197-213.

17. Orvidas LJ, Kasperbauer JL. Pediatric lymphangiomas of the head and neck. Ann Otol Rhinol Laryngol 2000;109: 411-21.

18. Bozkaya S, Ugar D, Karaca I, et al. The treatment of lymphangioma in the buccal mucosa by radiofrequency ablation: a case report. Oral Surg Oral Med Oral Pathol Oral Radiol Endod 2006; 102:e28-31.

19. Greene AK. Current concepts of vascular anomalies. J Craniofac Surg 2012;23:220-4.

20. Bisdorff A, Mulliken JB, Carrico J, et al. Intracranial vascular anomalies in patients with periorbital lymphatic and lymphaticovenous malformations. AJNR Am J Neuroradiol 2007;28:335-41.

21. Soltesz EG, Kim S, Laurence RG, et al. Intraoperative sentinel lymph node mapping of the lung using near-infrared fluorescent quantum dots. Ann Thorac Surg 2005;79:269-77.

22. Lee BB, Kim YW, Seo JM, et al. Current concepts in lymphatic malformation. Vasc Endovascular Surg 2005;39:6781.

23. Oosthuizen JC, Burns P, Russell JD. Lymphatic malformations: a proposed management algorithm. Int J Pediatr Otorhinolaryngol 2010;74:398-403.

24. Hogeling M, Adams S, Law J, et al. Lymphatic malformations: clinical course and management in 64 cases. Australas J Dermatol 2011;52:186-90.

25. Greene AK, Burrows PE, Smith L, et al. Periorbital lymphatic malformation: clinical course and management in $42 \mathrm{pa}-$ tients. Plast Reconstr Surg 2005;115:22-30. 